\title{
Mathematical and experimental modelling of flow of air-saturated water through a convergent-divergent nozzle
}

\author{
Jana Jablonská ${ }^{1, a}$ and Marian Bojko ${ }^{1}$ \\ ${ }^{1}$ VŠB-Technical University of Ostrava, Faculty of Mechanical Engineering, Department of Hydromechanics and Hydraulic Equipment, 17. \\ listopadu 15/2172, 70833 Ostrava - Poruba, Czech Republic
}

\begin{abstract}
In hydraulic elements an under-pressure is generated during fluid flow around sharp edges or changing the flow cross-section (e.g. for valves, switchgear, nozzles). In these locations air suction by leakages or release of air from the liquid during cavitation may occur. When flow modelling using classical mathematical model of cavitation at higher flow rates there is disagreement in the measured and calculated hydraulic variables before and behind hydraulic element. Therefore, it is necessary to use a mathematical model of cavitation applied to the three-phase flow (water, vapour, air). Nowadays it is necessary to look for mathematical approaches, which are suitable for quick engineering use in sufficiently precision numerical calculations. The article is devoted to theoretical investigation of multiphase mathematical model of cavitation and its verification using a laboratory experiment. At first case the k- $\varepsilon$ RNG turbulent mathematical model with cavitation was chosen in accordance [9] and was applied on water flow with cavitation (water and vapour) in a convergent-divergent nozzle. In other cases a solution of water flow with cavitation and air saturation was investigated. Subsequently, the results of mathematical modelling and experimental investigation focused on monitoring of air content and its impact on the value of hydraulic parameters and the size of the cavitation area were verified.
\end{abstract}

\section{Introduction}

Various hydraulic elements (throttle valve, switchgear, measuring equipment, etc.), which are characterized by constriction of cross section, are much discussed from point of the fluid flow. It is a specific multiphase flow of fluid mixtures with cavitation (vapour, liquid). If the pressure is equal to the saturated pressure, the cavitation occurs. Moreover, at this place the air can be added by due to the release from the liquid and suction by leakages $[13,14]$. The mixture is considered as the mixture of liquid, vapour and air.

Problem of mixture flow (gas - liquid) in convergent-divergent nozzle is described in the literature [5]. Authors determinate different values of the parameters such as pressure ratio, density ratio, Mach number and velocity ratio at different initial volume ratio. Measured values were taken from the experimental data while the calculated values came from theoretical relations. Error in the calculated and experimental values lay in the range of $(10-25) \%$ [5]. In reference [6] there are described experiments investigating the effects of air injection on the cloud cavitation of an oscillating hydrofoils. The article describes possibilities to reduce cavitation wear and noise levels during injection of air into the circuit.

The amount of air in the liquid has an influence on the size of the cavitation cloud and consequently on the distortion of the surfaces of various materials. Literature [7] generally described the influence of air and gases on cavitation (corrosion rate on the surface). The conclusions in the article show that increasing the oxygen level decreases the damage of the material surface. This decrease may be ascribed to the increased diffusion rate of air into cavitation bubbles during the growth cycle, leading to collapse cushioning [7].

Literature [8] studies effects of air content on cavitation and pressure fluctuations on the ship screw. From the results of the measurement, it is shown that air content has a great effect on the pressure fluctuations induced by the cavitating propeller. So air content has important effects on cavitation, especially on the bubble cavitation and the tip vortex cavitation and has little effect on the sheet cavitation range.

In this article we will deal with multiphase flow of water cavitation in the convergent - divergent nozzle (CD nozzle). From previous measurements authors know that at a given flow the pressure in the

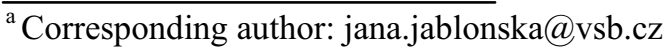


CD nozzle throat is decreasing to saturation pressure. To solve three-phase flow with cavitation the laboratory experiment for monitoring the size of the cavitation cloud and its changes at different air saturation was created. On the basis of this experiment the mathematical model was specified and the outcomes were verified with experimental data.

\section{Cavitation and aeration}

The fluid during cavitation is defined as multiphase mixture of liquid, vapour and air. Air is undissolved and takes the form of bubbles. The liquid containing of free air is not homogeneous medium (it is a suspension of gas in the liquid). Density of the liquid is significantly influenced by the content of gas, resulting in change in the dynamic properties of the fluid. The undissolved air exists in liquid always and like the vapour significantly affects the compressibility of the mixture. The undissolved air in the areas of low pressure combined with vapour creates a contiguous gas area. In the liquid there appears also dissolved air, which does not affect the compressibility of the fluid. Methods of detection of cavitation area and amount of gas are in figure 1 .

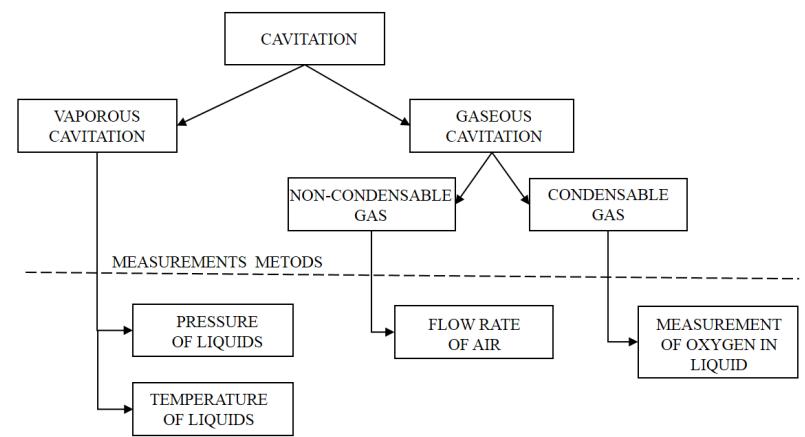

Figure 1. Specifications and methods of finding of cavitation area and gas quantities.

\section{Description of measurement}

The experimental equipment is located in the laboratories of the Technical University of Ostrava. The experimental equipment consists of a vertical centrifugal pump (figure 2, position 3), which is connected to water tank (figure 2, position 1). The centrifugal pump is driven by an electric motor (figure 2, position 4) with speed control by using the frequency converter (figure 2, position 5). Magnetic flowmeter is connected in the circuit below (figure 2, position 6). The circuit is supplemented by saturating air (figure 2, position 13 - 16), followed by transparent convergent divergent nozzle rectangular section - measured object (figure 2, position 8). Its basic dimensions are shown in figure 2. Sensors pressure (figure 2, position 9, 10) are disposed before and behind the CD nozzle. Time record of cavitation area (figure 2, position 17) is taken from each measurement using a camera.
Air is supplied to the circuit via the check valve and a mass flow regulator, which consists of a thermal mass flow sensor; control valve and accurate microprocessor PID (figure 2, position 13). Flow regulator adjusts quickly the required flow. The regulating device (figure 2, position 14) sets the desired volume flow rate of air, which then flows to the circuit.

The article deals with the influence of air amount on the size of the cavitation area at constant pump speed (the frequency converter is set at $26 \mathrm{~Hz}$ ). The first investigation was done for variant A without added air into the circuit and further for the variants $\mathrm{B}-\mathrm{E}$ with adding various amount of air. The size of the cavitation area was taken using the camera. Measuring of pressure before and after the CD nozzle, the volume flow rate of water (at $26 \mathrm{~Hz}$ ) is evaluated in table 2 .

Volume flow rates of air (see table 1) were recorded at each measurement. The measured values were averaged in time, and subsequently served as the boundary conditions for mathematical modelling. Operation range was limited by structural and material parameters of the equipment.

$\alpha_{\text {inlet }}$ is the volume fraction of air at the inlet of $\mathrm{CD}$ nozzle computed from measured pressure; $Y$ is the mass fraction of air; pressures are in absolute values, the atmospheric pressure is equal $101104 \mathrm{~Pa}$ to the day of measurement.

Visualization of the cavitation cloud for each measured variant was used for further evaluation. The term „cloud cavitation“ is used to refer to the process of coherent growth and collapse of clouds of cavitation bubbles [6].

Table 1. Indicated variants for different amount of air.

\begin{tabular}{|c|c|c|c|c|}
\hline \multirow{2}{*}{ variant } & $Q_{\text {air }}$ & $Q_{\text {m, air }}$ & $\alpha_{\text {inlet }}$ & $Y$ \\
\cline { 2 - 5 } & $\mathrm{ml}_{\mathrm{N}} \min ^{-1}$ & $\mathrm{~kg} \mathrm{~s}^{-1}$ & - & - \\
\hline $\mathrm{A}$ & 0 & 0 & 0 & 0 \\
\hline $\mathrm{B}$ & 100 & $4.87 \cdot 10^{-6}$ & $4.90 \cdot 10^{-4}$ & $1.43 \cdot 10^{-6}$ \\
\hline $\mathrm{C}$ & 300 & $1.46 \cdot 10^{-5}$ & $1.47 \cdot 10^{-3}$ & $4.30 \cdot 10^{-6}$ \\
\hline $\mathrm{D}$ & 400 & $1.95 \cdot 10^{-5}$ & $2.08 \cdot 10^{-3}$ & $6.10 \cdot 10^{-6}$ \\
\hline $\mathrm{E}$ & 500 & $2.43 \cdot 10^{-5}$ & $2.53 \cdot 10^{-3}$ & $7.39 \cdot 10^{-6}$ \\
\hline
\end{tabular}

Table 2. Measured value of water flow rate and pressure for different air flow rates.

\begin{tabular}{|c|c|c|c|}
\hline \multirow{2}{*}{ variant } & $\begin{array}{c}Q_{m, \text { water }} \\
\mathrm{kg} \mathrm{s}^{-1}\end{array}$ & $\begin{array}{c}p_{\text {inlet }} \\
\mathrm{Pa}\end{array}$ & $\begin{array}{c}p_{\text {outlet }} \\
\mathrm{Pa}\end{array}$ \\
\hline $\mathrm{A}$ & 3.393 & 188120 & 136539 \\
\hline $\mathrm{B}$ & 3.391 & 191155 & 136535 \\
\hline $\mathrm{C}$ & 3.380 & 192166 & 134516 \\
\hline $\mathrm{D}$ & 3.360 & 194189 & 133505 \\
\hline $\mathrm{E}$ & 3.325 & 195200 & 132493 \\
\hline
\end{tabular}




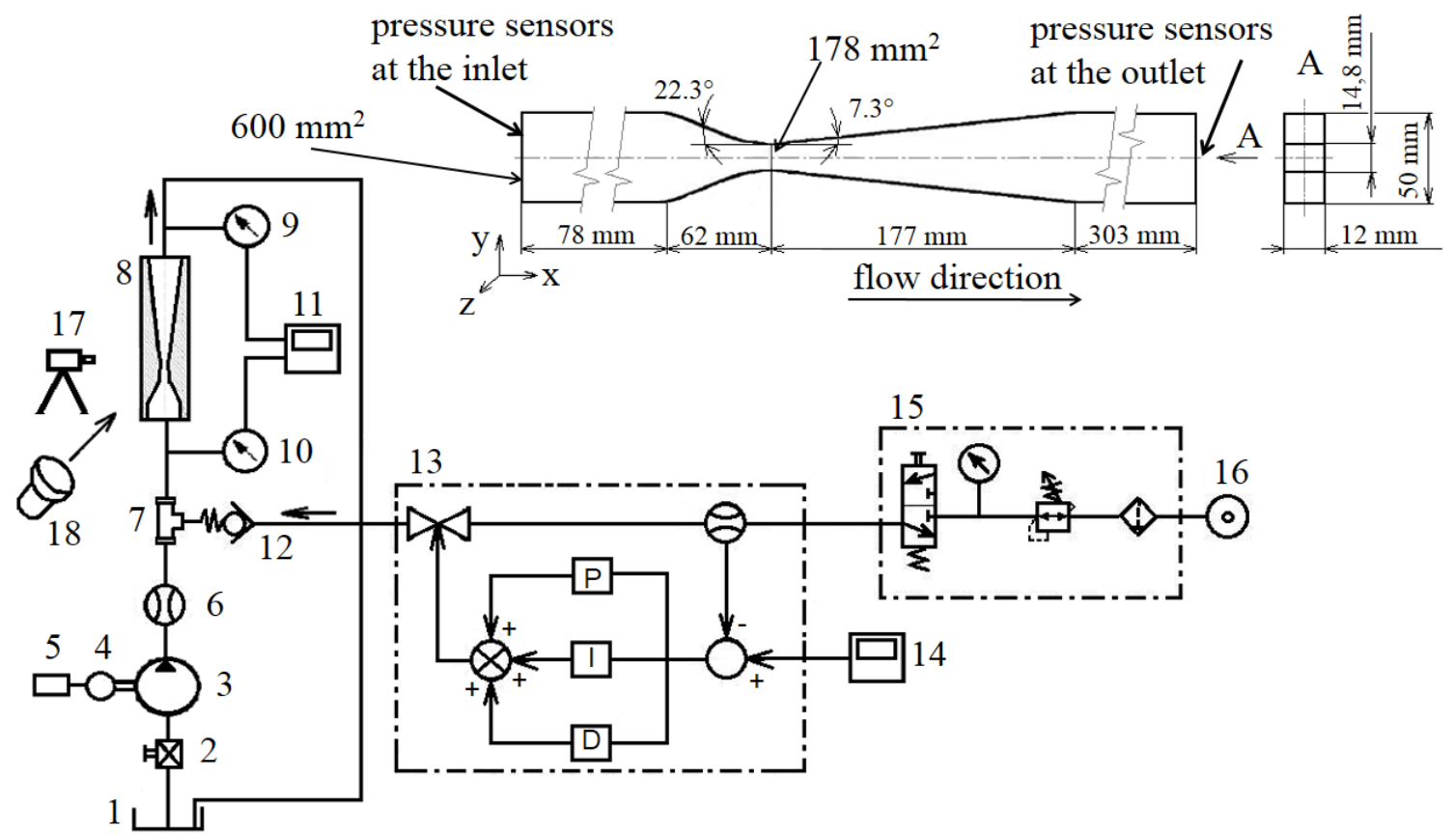

Figure 2. Circuit scheme - water and air segments $(1-$ tank, 2 - ball valve, 3 - hydro generator, 4 - motor, 5 - frequency converter, 6 - induction flowmeter, 7 - saturation with air, 8 - object measured, 9 -pressure sensor, 10 -pressure sensor, 11 evaluation equipment, 12 - one-way valve, 13 - mass flow controller, 14 - control unit, 15 - pressure regulating valve with a filter unit and a condensate separator, 16 - compressed air source with a pressure gauge, 17 - video camera, 18 - light source).

\section{Mathematical modelling}

Flow in the CD nozzle is turbulent, so equation of continuity and Navier-Stokes equation which take into account turbulence were solved. To the solution turbulence models were used ( $k$ - $\varepsilon$ standard, $k-\varepsilon$ RNG, $k-\varepsilon$ realizable, $k-\omega$ standard, $k-\omega \mathrm{SST}$, etc.). The system of differential equations was solved by finite volume method.

The mathematical model of turbulence cavitation takes into account the dynamics of gas bubbles, which is given generalized Rayleigh - Plesset equation for bubble dynamics (time-dependent pressure and size of bubbles) is

$$
\frac{p_{\text {vap }}(t)-p_{\infty}(t)}{\rho_{l}}=R_{b} \frac{d^{2} R_{b}}{d t^{2}}+\frac{3}{2}\left(\frac{d R_{b}}{d t}\right)^{2}+\frac{4 v_{l}}{R} \frac{d R_{b}}{d t}+\frac{2 S}{\rho_{l} R_{b}}
$$

for certain value of local far field pressure $p_{\infty}(t)$ the bubble radius $R_{b}(t)$ can be determined; $p_{\text {vap }}$ is saturation pressure; $\sigma$ is surface tension; $v_{l}$ is kinematic viscosity of liquid; $\rho_{l}$ is density of liquid; see $[1,2,3]$.

This differential equation has been too difficult to be applied into a multiphase flow model. Equation (1) was used in linearized form, for more details, please see [3, 4]

$$
\frac{d R_{b}}{d t}=\sqrt{\frac{2}{3} \frac{p_{\text {vap }}(t)-p_{\infty}(t)}{\rho_{l}}}
$$

Because the mathematical model should be taken into account evaporation of liquid and possibly air flow, the mathematical model is defined as multiphase flow model with cavitation by authors Schnerr and Sauer [2, 3, 4]. Cavitation model derives the exact expression for the mass transfer from liquid to vapour and condensation process equation for vapour volume fraction has the general form

$$
\frac{\partial}{\partial t}\left(\rho_{v a p} \alpha_{v a p}\right)+\frac{\partial}{\partial x_{j}}\left(\rho_{v a p} \alpha_{v a p} \bar{u}_{j}\right)=R
$$

where $\alpha_{v a p}$ is volume fraction of vapour; $\rho_{\text {vap }}$ is density of vapour; $R$ represents the vapour condensation or evaporation rate.

$$
R=\frac{\rho_{\text {vap }} \rho_{l}}{\rho}\left(\frac{\partial \alpha_{v a p}}{\partial t}+\frac{\partial\left(\bar{u}_{j} \alpha_{v a p}\right)}{\partial x_{j}}\right)
$$

where density of mixture can be defined by

$$
\rho=\alpha_{\text {vap }} \rho_{\text {vap }}+\alpha_{g} \rho_{g}+\left(1-\alpha_{\text {vap }}-\alpha_{g}\right) \rho_{l}
$$

and the volume fraction of vapour is expressed in this model using the number of bubbles $n_{b}$ in a unit of volume of liquid $[1,2]$.

$$
\alpha_{\text {vap }}=\frac{n_{b} \frac{4}{3} \pi R_{b}^{3}}{1+n_{b} \frac{4}{3} \pi R_{b}^{3}}
$$

after substituting

$$
\begin{gathered}
R=\frac{\rho_{\text {vap }} \rho_{l}}{\rho} \alpha_{\text {vap }}\left(1-\alpha_{\text {vap }}\right) \frac{3}{R_{b}} \sqrt{\frac{2}{3}} \frac{\left(p_{\text {vap }}-p\right)}{\rho_{l}} \\
R_{b}=\left(\frac{\alpha_{\text {vap }}}{\left(1-\alpha_{\text {vap }}\right)} \frac{3}{4 \pi} \frac{1}{n_{b}}\right)^{\frac{1}{3}}
\end{gathered}
$$

where $R$ is mass transfer rate; $R_{b}$ is bubble radius.

Also in this model, the only parameter which must be determined is the number of spherical bubbles per volume of liquid $n_{b}$. If you assume that no bubbles are created or destroyed, the bubble number density would be constant.

The main phase - water - contains small amount of non-condensable air, which can have significant influence on cavitation region due to compressibility of

\footnotetext{
${ }^{\mathrm{a}}$ Corresponding author: jana.jablonska@vsb.cz
} 
air by low pressure $[1,3,4]$. The calculation of the mixture density is modified as

$$
\frac{1}{\rho}=\frac{f_{\text {vap }}}{\rho_{\text {vap }}}+\frac{f_{g}}{\rho_{g}}+\frac{1-f_{\text {vap }}-f_{g}}{\rho_{l}}
$$

Non condensable gas density $\rho_{g}$ is calculated by equation of state as

$$
\rho_{g}=\frac{M p}{R T}
$$

Volume fractions of non-condensable gas and liquid are modified as

$$
\alpha_{g}=f_{g} \frac{\rho}{\rho_{g}}, \quad \alpha_{l}=1-\alpha_{v a p}-\alpha_{g}
$$

where $\alpha_{\text {vap }}$ is volume fraction of vapour; $\alpha_{g}$ is a volume fraction of gas; $f_{g}$ is gas mass fraction.

\section{Numerical modelling of flow in convergent divergent nozzle rectangular section}

Task is solved as a three-dimensional, symmetrical area. The geometry of the modelled area identical with the measuring, grid and boundary conditions are shown in figure 3., input and output are in places located pressure sensors. The grid was created in the program Gambit, has 502500 hexahedrons cells. Due to assessing the size of cavitation area there is created in the model the evaluation plane with a constant distance of $0.01 \mathrm{~m}$ from the CD nozzle throat.

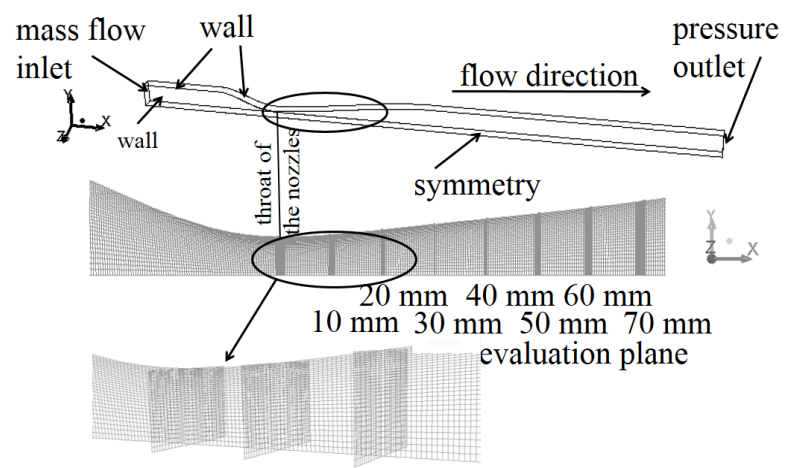

Figure 3. The geometry of the modelled area and boundary conditions.

$K-\varepsilon$ RNG two-equation turbulent model and nonequilibrium wall functions were chosen based on the Reynolds number and recommendations references [10, $11,12]$. The task was calculated as the time dependent with a time step $1 \cdot 10^{-5} \mathrm{~s}$, due to dynamics of cavitation cloud. Gravity acceleration in the negative direction of the $\mathrm{x}$-axis was considered due to the location of the $\mathrm{CD}$ nozzle in the measurement.

The physical properties are defined for water and vapour as constant. Water density is $998.2 \mathrm{~kg} \mathrm{~m}^{-3}$ and viscosity of water is $0.001003 \mathrm{~Pa} \mathrm{~s}$. Vapour density is $0.017 \mathrm{~kg} \mathrm{~m}^{-3}$ and viscosity of vapour is $1.34 \cdot 10^{-5} \mathrm{~Pa} \mathrm{~s}$. Air density is defined as ideal-gas and viscosity of air is $1.789 \cdot 10^{-5} \mathrm{~Pa}$ s. Values of saturation vapour pressure and bubble number density $10^{+13}$ are necessary to define for the cavitation model. Saturation vapour pressure for a given constant temperature $25^{\circ} \mathrm{C}$ (isothermal condition) is $2340 \mathrm{~Pa}$. The measured data were averaged for the determination boundary conditions.

Table 3. Boundary and cavitation conditions.

\begin{tabular}{|c|c|}
\hline inlet - mass flow inlet* & mass flow rate \\
\hline water & see table 2, variant A - E \\
\hline vapour & $0 \mathrm{~kg} \mathrm{~s}^{-1}$ \\
\hline air & see table 1, variant A - E \\
\hline outlet - pressure outlet & see table 2, variant A - E \\
\hline cavitation conditions & Schnerr - Sauer model \\
\hline saturation vapour pressure & $2340 \mathrm{~Pa}$ \\
\hline bubble number density & $10^{+13}$ \\
\hline
\end{tabular}

* Only half of the flow rate is necessary to insert into numerical simulation because of the symmetry task.

Boundary conditions are defined for different variants of the volume fraction of gas (air) (see table 1). The average mass flow of water, air at the inlet and the average outlet pressure is measured.

Testing of the turbulent model $k-\varepsilon$ (standard, RNG, realizable) and $k-\omega$ (standard, SST) is presented in the publication [9]. Models were tested for the given geometry and the grid, but it showed some anomalies that are not compatible with the experiment. Model $k-\varepsilon$ RNG is appropriate to use. This which follows the recommendations of the literature $[10,11,12]$ and also from testing of the models. Evaluation of testing was performed by comparing of the modelling results and of the measurements.

Grid quality was evaluated by calculation, i.e. task was calculated with larger and smaller number of cells. Deviation of the average pressure value at the inlet was 1 $\%$. The parameter $y+$ was also evaluated for chosen model and grid, its value is $30-60$, so the grid is suited.

\section{Comparison of modelling and measurement}

On figure 4 the cavitation cloud is visualized for each variant of the input boundary conditions. The figure shows that the area of vapour is approximately still the same. The cavitation cloud increases with increasing amount of air that is added to the circuit.

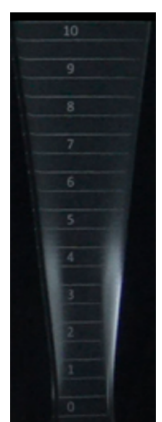

A

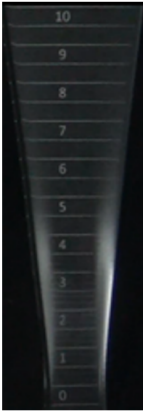

B

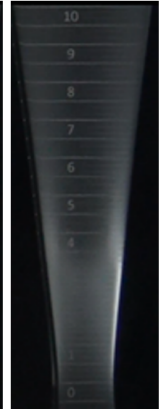

C

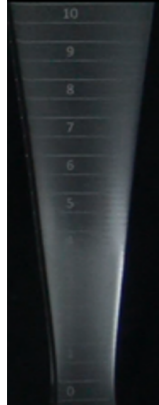

D

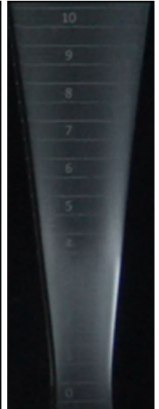

E
Figure 4. Visualization of measurement. 


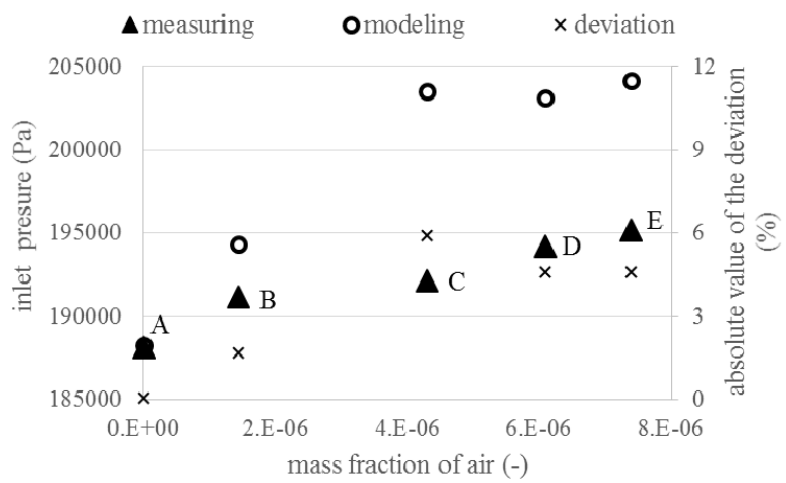

Figure 5. Inlet pressure dependence on the mass fraction of air.

Dependence of measured and mode led inlet pressure vs. mass fraction of air is evaluated on figure 5 . Modelling and measurement error is $6 \%$ (compared to the article mentioned in the introduction [5] where the error was $(10-25) \%$. It is very good result.

From the graphical dependence of the volume fraction of vapour, air, vapour and air, and water on a mass fraction of air in the whole volume of the CD nozzle it is evident that the volume fraction of water decreases with increasing undissolved air. The volume fraction (or amount) of vapour can be evaluated as constant in whole the volume of the $\mathrm{CD}$ nozzle. It follows that the volume fraction of water (and consequently the size of the cavitation area) depends on the amount of undissolved air in the liquid.

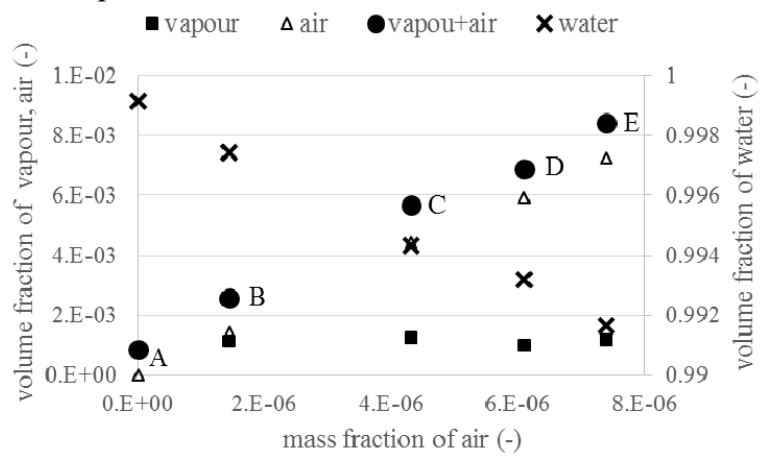

Figure 6. Dependence of volume fraction of vapour, air and water in the whole volume of the CD nozzle vs. mass fraction of air.

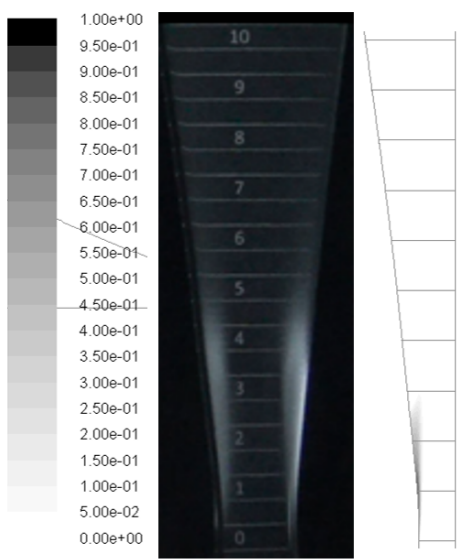

Figure 7. Variant A - comparison of the size of the cavitation cloud gained by experiment and modelling of water and vapour.

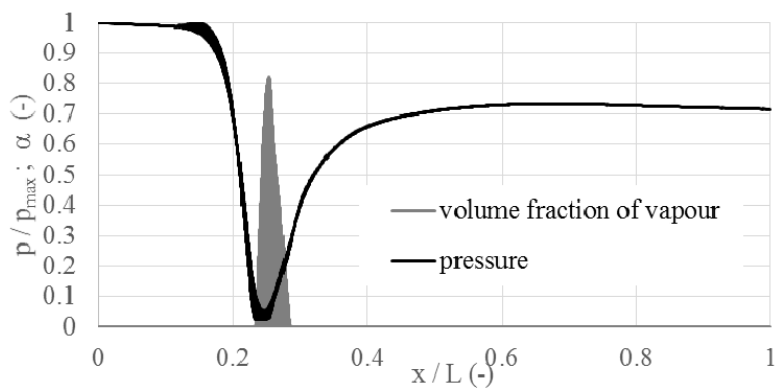

Figure 8. Variant A - distribution of dimensionless pressure ratio and volume fraction of vapour along the wall.

On figure 7 there is apparent good consensus of the size of the cavitation cloud when modelling variant $\mathrm{A}$ (water and vapour).

Change of pressure ratio along the length of the $\mathrm{CD}$ nozzle is apparent on the figure 8 . Pressure minimum in the throat of the CD nozzle has value of $2340 \mathrm{~Pa}$ (saturated vapour pressure). At this place vapour is observed. The amount of vapour volume is declining with increasing pressure (along the length of the CD nozzle).

Vapour area is depicted at the left of figure 9 and vapour and air area is depicted at the right. When comparing figure 7 and figure 9 it is evident that the area of vapour was not changed. Increasing size of the cavitation cloud is in accordance with the growing amount of added air. Modelling shows that the vapour volume fraction changes only slightly depending on mass fraction of added air (see figure 6). The size of the area depends on the amount of undissolved air in the liquid.

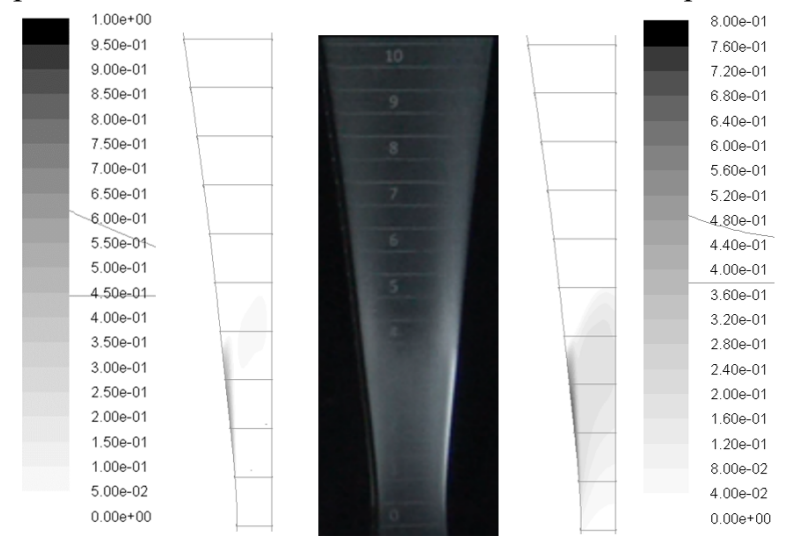

Figure 9. Variant E - comparison of the size of the cavitation cloud gained by experiment and modelling of water, vapour and air.

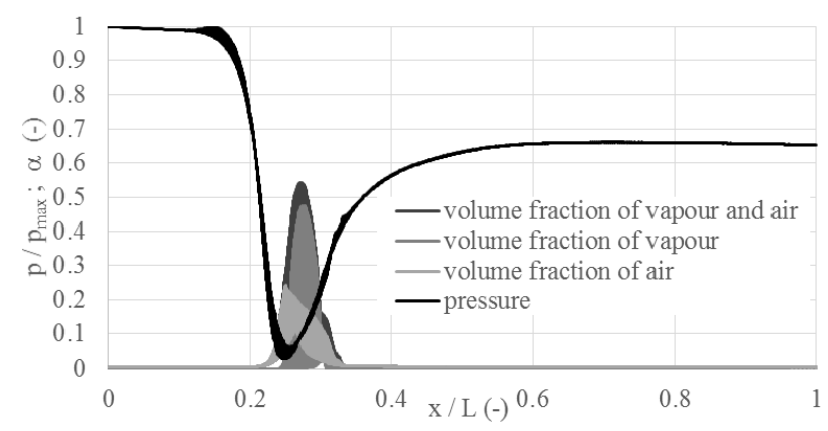

Figure 10. Variant $\mathrm{E}$ - distribution of pressure ratio and volume fraction of vapour along the wall. 
Figure 10 shows that variation of pressure ratio along the length of the CD nozzle is not changed in comparison with the variant A but volume fraction of vapour decreased and the length of the area vapour and air is increased. The amount of vapour remains approximately constant in comparison with variant $\mathrm{A}$, see figure 11, where the lower (dark) part of the column indicates the length of the cavitation vapour area. The whole length of the column indicates the size of the cavitation area of vapour and air.

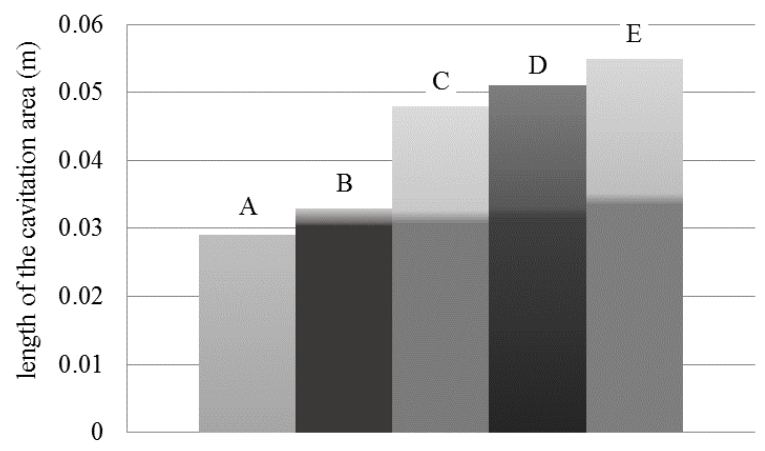

Figure 11. Length of the cavitation area on the wall (variant A E).

\section{Conclusion}

The work is devoted to theoretical investigation of multiphase mathematical model of cavitation at higher flow rates where there is disagreement in the measured and calculated hydraulic variables before and behind hydraulic element. The article deals with the specification of the general mathematical model of flow with cavitation and its application to multiphase flows (water, vapour, air). The results were verified with experiments aimed on water flow with cavitation in the convergentdivergent nozzle and the air saturation.

- $\quad$ RNG k- $\varepsilon$ mathematical model of turbulent flow with cavitation was defined for Reynolds number at the input about 100 000. Flowing medium was specified as a mixture of incompressible liquid (water) and vapour. Air was considered as a next compressible phase.

- The CD nozzle resistance was increased by adding air into the hydraulic circuit, therefore measured parameters as flow rate and pressure at the inlet and outlet at constant speed of the pump are changed. Hydraulic values obtained from experiment and numerical calculation differed by less than $6 \%$.

- Size of cavitation cloud depended on the amount of undissolved air, increasing amount of air increases the size of the cavitation cloud. This was confirmed by visualization and numeric calculation.

- Significant numerical result is that the amount of vapour is not dependent on the amount of air in the liquid.

We can conclude that the commonly used mathematical model of cavitation should be extended to phase of air when applied to liquid flow in hydraulic elements at higher operating parameters.

\section{References}

1. Fluent 15 - User's guide. [Online]. Available to from: <URL: http://sp1.vsb.cz/DOC/Fluent_6.1/ $\mathrm{html} / \mathrm{ug} /$ /main_pre.htm>.

2. M. Kozubková, Numerické modelováni proudění FLUENT I. [Online]. Available to from: <URL: http://www.338.vsb.cz/seznam.htm>. (2005)

3. M. S. Plesset, R. B. Chapman, J. of Fluid Mechanics, 47 (02), (1971).

4. A. K. Singhal, M. M. Athavale, H. Li, Y. Jiang, J. of Fluids Engineering, 124 (3), (2002).

5. A. Ullah, M. Nadeem, A. N. Ahmed, Emerging Technologies, 2009. ICET 2009. International Conference on. IEEE, (2009).

6. G. E. Reisman, M. E. Duttweiler, C. E. Brennen, ASME Fluids Engineering Division Summer Meeting, (1997)

7. J. G. Auret, O. F. R. A. Damm, G. J. Wright, F. P. A. Robinson, Tribology international, 26 (6), (1993).

8. J. M. Ye, Y. Xiong, L. I. Fang, S. Q. Chen, J. of Hydrodynamics, Ser. B, 22 (5), (2010).

9. J. Dobeš, M. Kozubková, EPJ Web of Conferences 67, p. 02019. EDP Sciences. (2014)

10. S. Martynov, D. Mason, M. R. Heikal, S. S. Sazhin, M. Gorokhovski, International Heat Transfer Conference 13. (2006)

11. P. Roosen, O. Unruh, M. Behmann, Report of the Institute for Technical Termodynamics. (1996)

12. E. Winklhofer, E. Kull, E. Kelz, A. Morozov, ILASS-Europe 2001, 17 International Conference on Liquid Atomization and Spray Systems. (2001)

13. L. Hruzik, M. Vasina, A. Burecek, EPJ Web of Conferences 45, 01041 (2013)

14. J. Jablonská, M. Kozubková, J. of Computational Multiphase Flows, 7 (2), (2015)

\section{Acknowledgements}

The work presented in this paper was supported by a grant SGS "Zkoumání dynamiky tekutinových systémů" SP2015/95. 